\title{
Ubiquitous Organizational Information Service Framework for Large Scale Intelligent Environments
}

\author{
Kwang-il Hwang ${ }^{1}$, Won-hee Lee ${ }^{1}$, Seok-hwan Kim ${ }^{1}$, \\ Doo-seop Eom ${ }^{1}$, and Kyeong Hur $^{2}$ \\ ${ }^{1}$ Department of Electronics and Computer Engineering, Korea University, \\ \#1-5ga, Anam-dong, Sungbuk-gu, Seoul, Korea \\ \{brightday, wangpepe, sukka, eomds\} afinal.korea.ac.kr \\ ${ }^{2}$ Department of Computer Education, \\ Gyeongin National University of Education \\ khur@ginue.ac.kr
}

\begin{abstract}
In this paper, we introduce a concrete, practical Ubiquitous Organizational Information (UOI) service framework, providing novice users intelligent and useful services with respect to the environment. The UOI framework based on the sensor networks is composed of 3-level hierarchical network architecture. To provide a rich array of services, the modular software framework and foundation software are designed and implemented on our hardware prototype. We define three representative UOI services and illustrate each service flow operating on the proposed UOI network. In addition, we describe some details in the implementation of a distributed UOI network on the UOI test-bed.
\end{abstract}

Keywords: Distributed Sensor Networks, Embedded Systems, Intelligent Environment, and Ubiquitous Computing.

\section{Introduction}

To coincide with the grand pervasive computing vision, everyday computing spaces will need to become a component of the user's normal background environment, gradually becoming more ubiquitous in nature. Mark Weiser first initiated the notion of Ubiquitous Computing at Xerox PARC [1], who envisioned in the upcoming future, ubiquitous interconnected computing devices that could be accessed from any location, used effortlessly, and operate unobtrusively, even without people's notice of them, just as that of electricity or telephones are used today.

Many researchers define an intelligent environment, as one of the most representative applications of Ubicomp, as an augmented spacious environment populated with many sensors, actuators and computing devices. These components are interwoven and integrated into a distributed computing system, capable of 
perceiving context through sensors, to execute intelligent logic on computing devices and serve occupants by actuators. This intelligent environment is extending its range from a users' personal room or classroom, to a large house or building.

Let us suppose the following situation. We visit an unfamiliar environment, which presents a wide area, such as an amusement park, university campus, or large building. Confusions often arise when finding the location of something or where to travel next. Furthermore, the use of certain facilities may be desired or someone in the organization may need to be found. Such users' needs will be satisfied with an intelligent service involving information regarding the organization. Our UOI service framework is designed to provide such an intelligent service to users, especially for large scale environment. In this paper, the presented UOI service framework presents more concrete and practical way to create intelligent environment.

The rest of this paper is organized as follows. We first outline several researches related to the intelligent environment. Then, the UOI framework is presented, which is composed of three major components. Subsequently, the UOI service flow through the distributed UOI network is illustrated. Our hardware prototype and UOI foundation software operated on the prototype is also introduced. Lastly, details in the implementation of a distributed UOI network are described. A conclusion is provided with a description of future work.

\section{Related Work}

There have been substantial researches relating to the construction of ubiquitous environments.

Cooltown [2] and the associated CoolBase infrastructure aim to give people, places, and things a Web presence. Although Web technology is proven and widely available, it has inherent complexity, since, to be connected to the Web, a fully supported TCP/IP stack and system capable of running the relatively heavy software is required.

Projects, such as Gaia [8], Microsoft Easy Living [3], and CORTEX [9], aim to develop an infrastructure to support augmented environments in a fairly broad sense. They provide basic abstractions and mechanisms for coping with the dynamics and device heterogeneity of pervasive computing environments. There is quite a large difference between the projects and the framework presented in this paper. While they provide application models that are still rather generic, our work supports a rather specific application model.

In such a sense, PiNet [10] is the most similar to the presented model in that the final goal is to provide an organizational information service to users. However, the work in this paper is distinguished from PiNet primarily in the uses of sensor networks. In contrast to PiNet using a global cellular network as an infrastructure, the UOI adopts distributed sensor networks. In our research, service network infrastructure and service framework based on sensor networks, are more emphasized, instead of focusing on user perception or virtual reality as in [4 - 7]. 


\section{Architecture of UOI Framework}

The UOI service framework infrastructure is based on distributed sensor networks. It is assumed that the environment is covered with innumerable tiny sensor nodes, which are extremely limited in power, processing, and memory resources. The sensor nodes are called access nodes, since they are used as access points connecting users to a UOI network. Each node is aware of its own location information by manual planning or other localization algorithms, and possesses the ability to communicate with user's devices via short range wireless communication. The UOI service infrastructure using sensor networks, not global networks such as cellular networks or GPS, presents advantages as follows.

- Guarantee of freshness with respect to the dynamics of information

- Security

- Service charge issues regarding information use

Firstly, the update of information with respect to the change of an organizational structure or service will be achieved faster and easier through a scalable UOI framework. Secondly, organizational information will be safer from outside networks. Lastly, users are allowed to use the service without any extra communication charge. In addition to these advantages, compared to WLAN networks, the UOI network architecture provides more elaborate location information and increases efficiency in the use of facilities through sensors and actuators. The UOI network architecture also enables localized information processing and fusion, by clustering regionally adjacent access nodes.

\subsection{Key Features of UOI Framework}

The presented framework is designed to efficiently provide UOI services. Such UOI framework includes the following array of features.

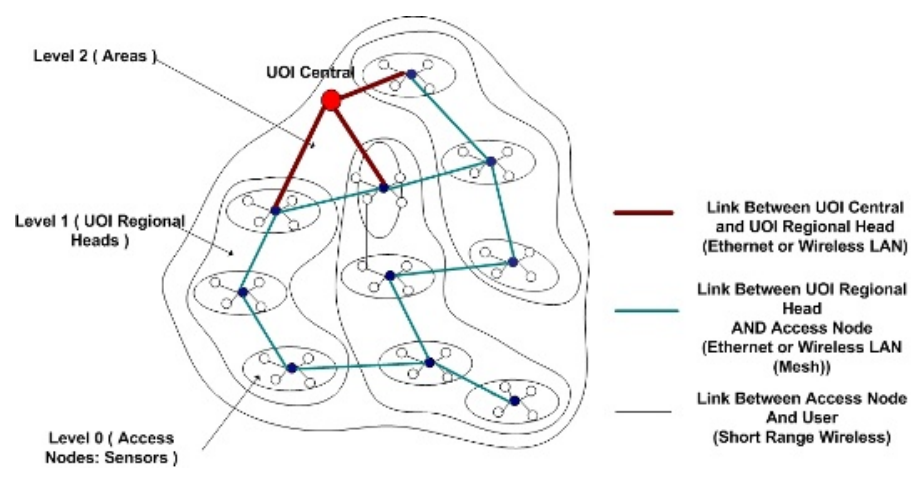

Fig. 1. Hierarchical UOI network architecture based on distributed sensor networks 
Hierarchical architecture: As shown in Fig. 1, the UOI framework is composed of three-tier architecture, more specifically, access node level, regional head level, and UOI central. Regionally adjacent nodes form a cluster in which a cluster head node is responsible for managing its own cluster member nodes. In addition, the clusters form a network of tree-based or mesh-based topology to communicate with each other. The clusters are also connected to the UOI central, which operates similarly to a central server. This hierarchical architecture of UOI framework makes it possible to localize information in a cluster, and reduces traffic by using aggregation and fusion within a cluster head. This feature is also useful when the environment is managed regionally.

Property-based naming and information-centric routing: The UOI platform uses property-based naming, similar in concept to naming in the Directed Diffusion [11], not global ID such as IP address or MAC address, as a node identifier. Each node has an inherent name related to its property such as location or sensing ability. For example, 'East2 Floor1' means the node is the $2^{\text {nd }}$ node from UOI regional head of the $1^{\text {st }}$ floor to east. In addition, 'Tb21 KoreanRest1' will be the 21 th table number of a Korean restaurant in a huge amusement park. Information-centric routing is also enabled by virtue of the property-based naming, which is different from addresscentric routing.

Distributed querying and tasking: In the UOI framework, user's service request is translated into a query to be flooded to the UOI network. The query is injected in each access node and the UOI regional head via the UOI hierarchy. In each node, the query generates a corresponding task, operating on the UOI foundation software. This distributed tasking demonstrates some results with respect to the query and only the nodes, which have data matched with the query, can report matched information to UOI central. This feature reinforces the distributed information processing ability in the UOI network, in contrast to other global or centralized networks.

Transparent services: The UOI framework provides transparent services to users. Users only request a service with their device, and specific actions for configuration or registration are not required. When entering an area covered with a UOI network, the user is expected to turn on the device and be automatically connected. No configuration changes are necessary as the user moves from one site to another. The network needs no pre-knowledge regarding the device attempting to connect to it.

\subsection{Components for UOI Service Framework}

The UOI network infrastructure is composed of three distinguished components, Access node, UOI regional head, and UOI central, as shown in Fig. 2. These independent components play an important role in building a UOI service framework with a hierarchical architecture.

Access node is the most basic component, allowing users to access the UOI infrastructure. This component is composed of UOI foundation software, Query Translation Unit, Task Manager Unit, User Interaction Manager, and Location Management Unit. 


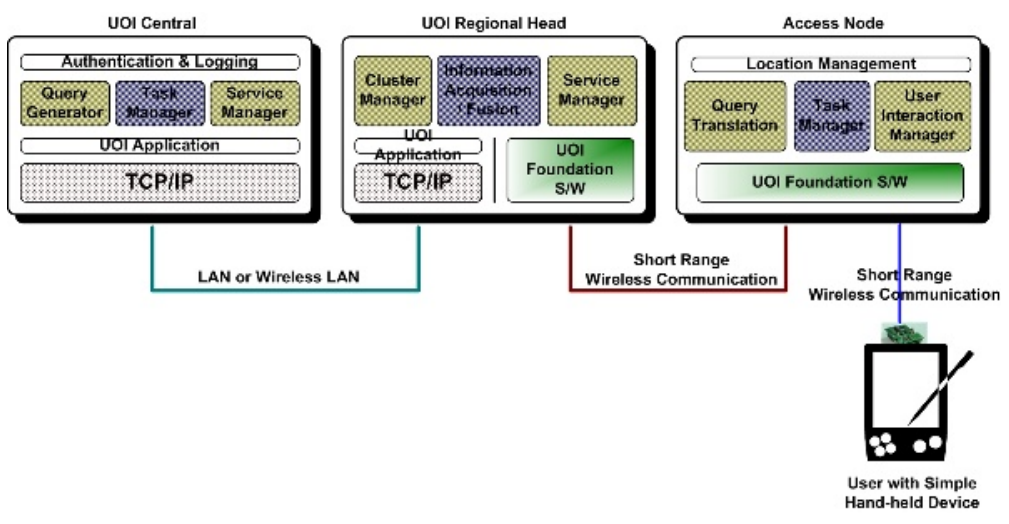

Fig. 2. Components for UOI service framework

UOI Regional Head as a cluster head is responsible for managing access nodes in its own cluster, and operates as a gateway between access nodes and UOI central. The UOI head includes two independent networks software: TCP/IP for connecting to UOI central by LAN or WLAN, and UOI foundation software for managing cluster members. In addition, the role of UOI head is performed with UOI application, Cluster Manager Unit, Information Acquisition and Fusion Unit, and Service Manger Unit.

UOI Central represents component of the highest level in the UOI service hierarchy. It plays an important role in generating queries with respect to user's service request, managing tasks and services and authenticating users and logging. The works are performed with the Query Generation Unit, Task Manager, Service Manager, and Authentication Unit.

\subsection{Organizational Information Services Through Distributed UOI Networks}

Users entering an unfamiliar environment want to get some organizational information and be available to freely use various facilities without pre-knowledge in the organization. Furthermore, users do not want to manually configure or register themselves to use organization services. We summarize the UOI services fulfilling such user's requirements into the following service category.

Location guide service: First of all, the most basic service offered to novice users is location guidance. Generally, guiding services using GPS are the most common. However, in GPS, the service with high resolution is not guaranteed. In addition, GPS is difficult to be used for indoor location systems, such as large buildings.

Compared with the GPS service, our UOI framework provides more reliable location guide service with higher resolution through distributed UOI networks. Figure 3 shows the procedure of location guide services in the UOI framework. As shown in Fig. 3, the nearest access node listens to user's service request and then 
sends the request message to the UOI regional head. After successful authentication between the head and central, the user's service request at UOI central is transformed into a corresponding query and the query is flooded over the network. As soon as the query is received, each node executes a corresponding task from the query. The nodes with matched data send the response upstream, immediately. UOI central performs aggregation, fusion, and result generation during a given period. The result is delivered to the UOI regional head of the targeted node.

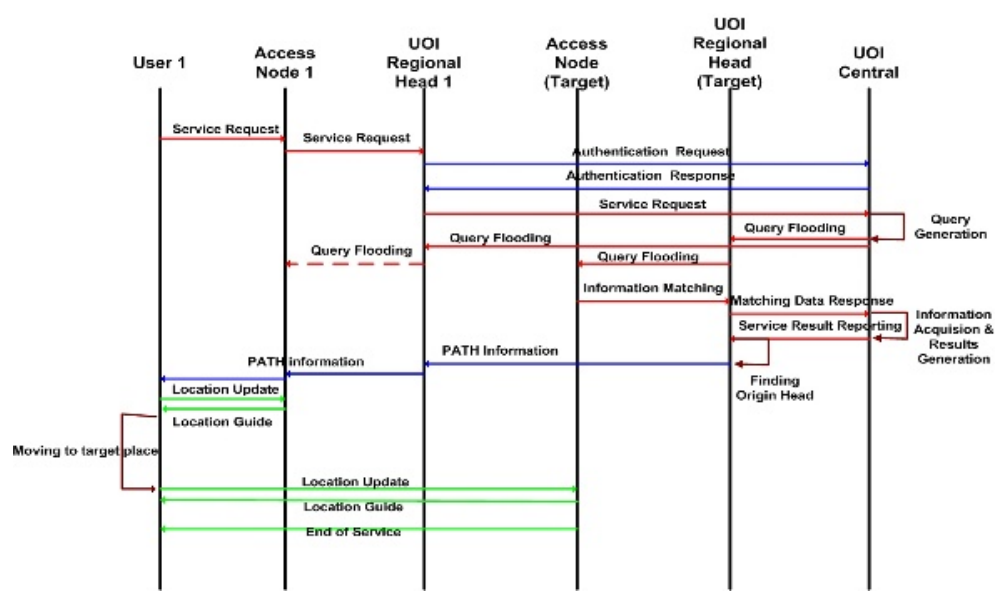

Fig. 3. Process procedure of location guide service in UOI framework

The targeted head retrieves the source head to establish an exclusive link between the two regional heads. The targeted head sends PATH Information to the source head, including all path information from the target to source. Note that this logical path is regarded as a reliable physical path, since we assume the environment is densely covered with sensor nodes. Simultaneously, all access nodes, included on the path between two heads, execute the location guide task and process location update messages from the user. Now, a user can view the location where the user wants to go, which will be displayed on his or her device. The user's device sends location update message periodically and each access node guide the user by responding with a location guided message.

Use of facilities in the organization: One of the representative intelligent services is to allow users to use various facilities without prior knowledge in the organization. For example, in an amusement park, we want to find a specific amusement facility. However, generally we must wait for a long time just for the short instance actually using the service. This delay may annoy users, but they cannot help using the service.

However, in the UOI service framework, users do not have to wait for a long line. Instead of waiting until the user's order comes, users simply make a reservation for 
the use of the facility that the user wants while enjoying other amusement facility. After the reservation, when the user's service is available, the user is alarmed through the UOI network. Figure 4 shows the detailed flow for user to use facilities in the UOI framework. The service is divided into four phases. The first phase consists of service request and authentication, and second phase includes the discovery of service access points and a status result report. These two phases are almost similar in a location guide service. The third phase is user's reservation to use the service. The last is service reservation delivery and result reporting. All the processes are accomplished through distributed UOI networks and completely transparent to users.

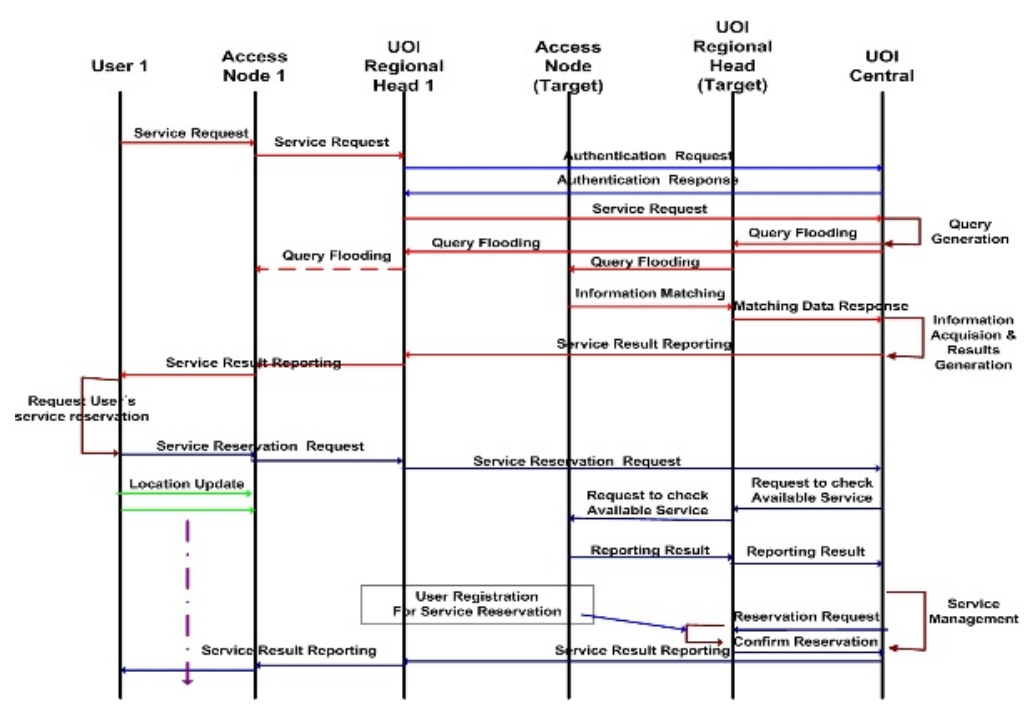

Fig. 4. Process procedure for use of facilities in UOI framework

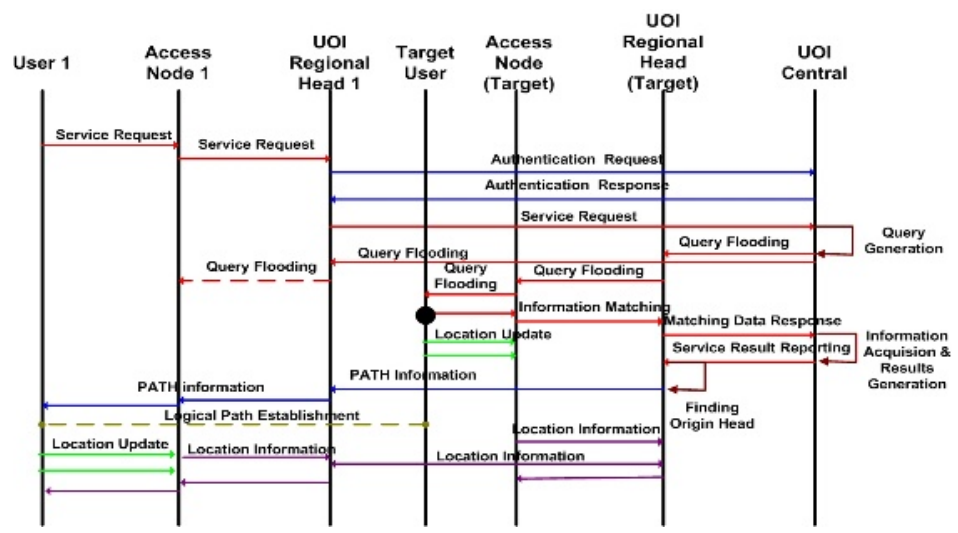

Fig. 5. Process procedure for people search 
People search: The other required service involves finding people within an organization. This is useful to find out a missing child's location or the location of someone who is not available to use a phone in the organization. In addition, this service is used to retrieve the corresponding user, who is reserved to use a facility, to notify that the service is now available. For this service, general steps are similar to others but it is outstanding that the exclusive logical path between the user, who requests the service, and target user is established. Note that for concurrent mobility support, location managements for both are performed as shown in Fig. 5.

\section{UOI Network Implementation}

In order to implement the proposed UOI service framework, a UOI test-bed as shown in Fig. 8, was developed. As an access node, a scalable prototype having a very compact size of a square inch was developed as shown in Fig. 6. Also, Figure 7 illustrates the event-driven UOI foundation software operated on the prototype.

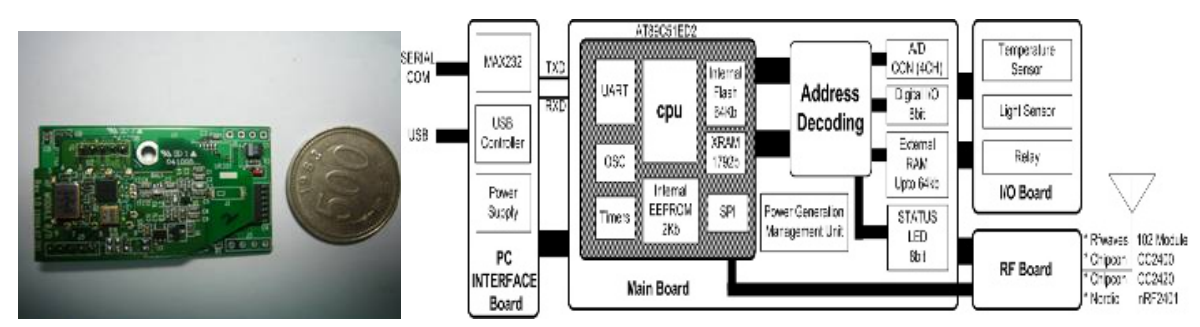

Fig. 6. Prototype for UOI access nodes and user interface

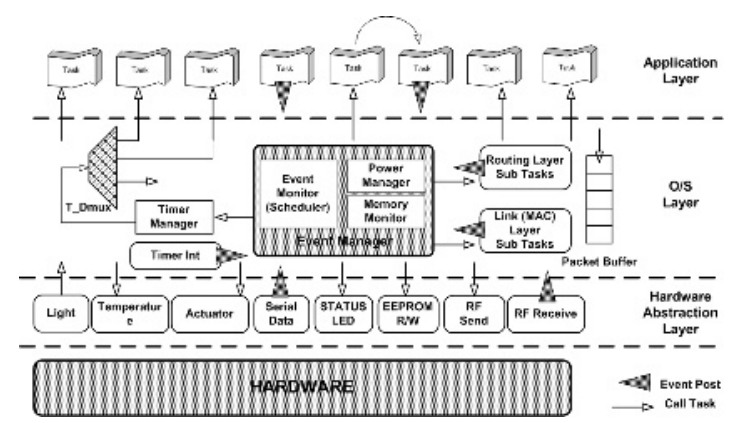

Fig. 7. UOI foundation software architecture

The Test-bed is composed of access nodes, which is built-in UOI foundation and framework software on our prototype, regional heads, and UOI central. For higher performance of Regional heads, an embedded system with Intel's Xscale MCU 
(PXA255) was used as a UOI regional head and UOI central software was executed on a desktop PC. The deployment of each component is as shown in Fig. 8, including two users and two service points.

In the test-bed, three kinds of UOI services that we described in Section 3.3 were experimented: in the first experiment, user A is guided to service point 2 through the UOI service. Secondly, user A and B reserved an available arbitrary seat in service point (room) 1 and 2, respectively, and then were guided to the corresponding service point. In the final experiment, mobile user A tracked mobile user B, continuously.

Initial architecture design was revised from a more practical sense through the experiment. After experiencing innumerable trials and errors with the experiment, measurable improvements were made to the complete UOI service framework architecture.

\section{Conclusion and Future Works}

We proposed a concrete, practical Ubiquitous Organizational Information (UOI) service framework, providing novice users intelligent and useful services respecting the environment. The UOI framework consists of hierarchical network architecture, based on distributed sensor networks. To provide a rich array of services, the designed UOI framework and foundation software are implemented on our hardware prototype. In addition, representative UOI services were tested on the UOI test-bed.

Currently, we are investigating to extend the kind of services and improve the quality of services.

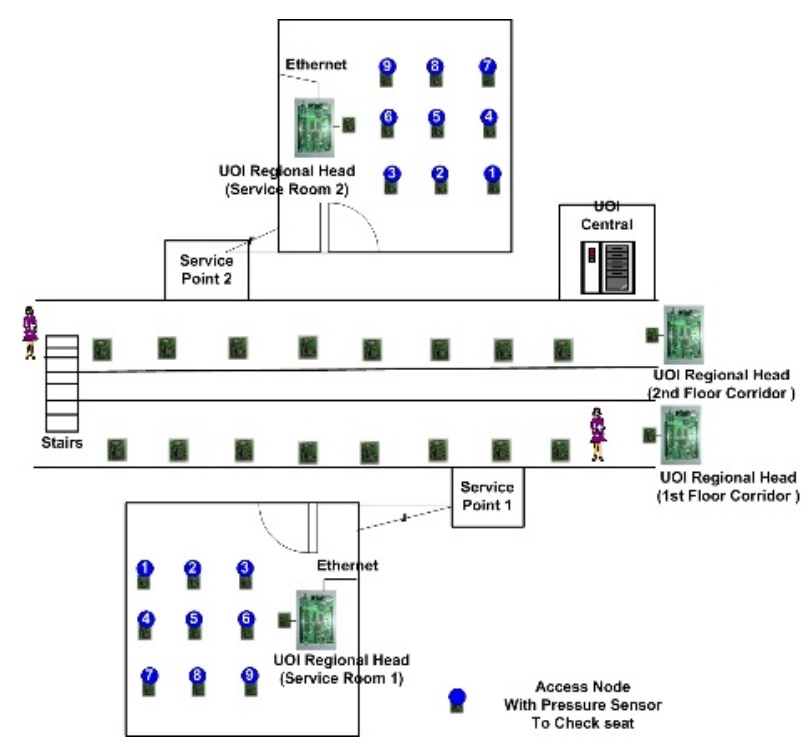

Fig. 8. Test-bed environment for implementation of UOI service framework 


\section{References}

1. M. Weiser, "The Computer for the $21^{\text {st }}$ Century," Scientific American, September, 1991.

2. HP Cooltown. http://www.cooltown.com/cooltown/

3. Microsoft Easy Living. http://research.microsoft.com/easyliving/

4. MIT AI Lab AIRI Project, 2000. http://aire.csail.mit.edu/

5. Georgia Tech, Aware Home Research Initiative (AHRI) Project, http://www.cc.gatech.edu/fce/ahri/

6. CMU Project AURA. http://www-2.cs.cmu.edu/ aura/

7. Sony Augmented Surfaces. http://www.csl.sony.co.jp/person/rekimoto/as/

8. M. Roman, C. Hess and R. Campbell, Gaia: An OO middleware infrastructure for ubiquitous computing environments, ECOOPOOOSWS 2002, 2002.

9. P. Verissimo, V. Cahill, A. Casimiro, K. Cheverst, A. Friday and J. Kaiser, CORTEX: Towards supporting autonomous and cooperating sentient entities, European Wireless 2002, Florence, Italy (February2002).

10. Carmel, B. et al., "PiNet: Wireless Connectivity for Organizational Information Access Using Light-weight Handheld Devices," IEEE Personal Communications, Aug. 2001.

11. C. Intanagonwiwat, R. Govindan, and D. Estrin, "Directed diffusion: A scalable and robust communication paradigm for sensor networks," 6th ACM/IEEE Mobicom, 2000. 